\title{
Editorial
}
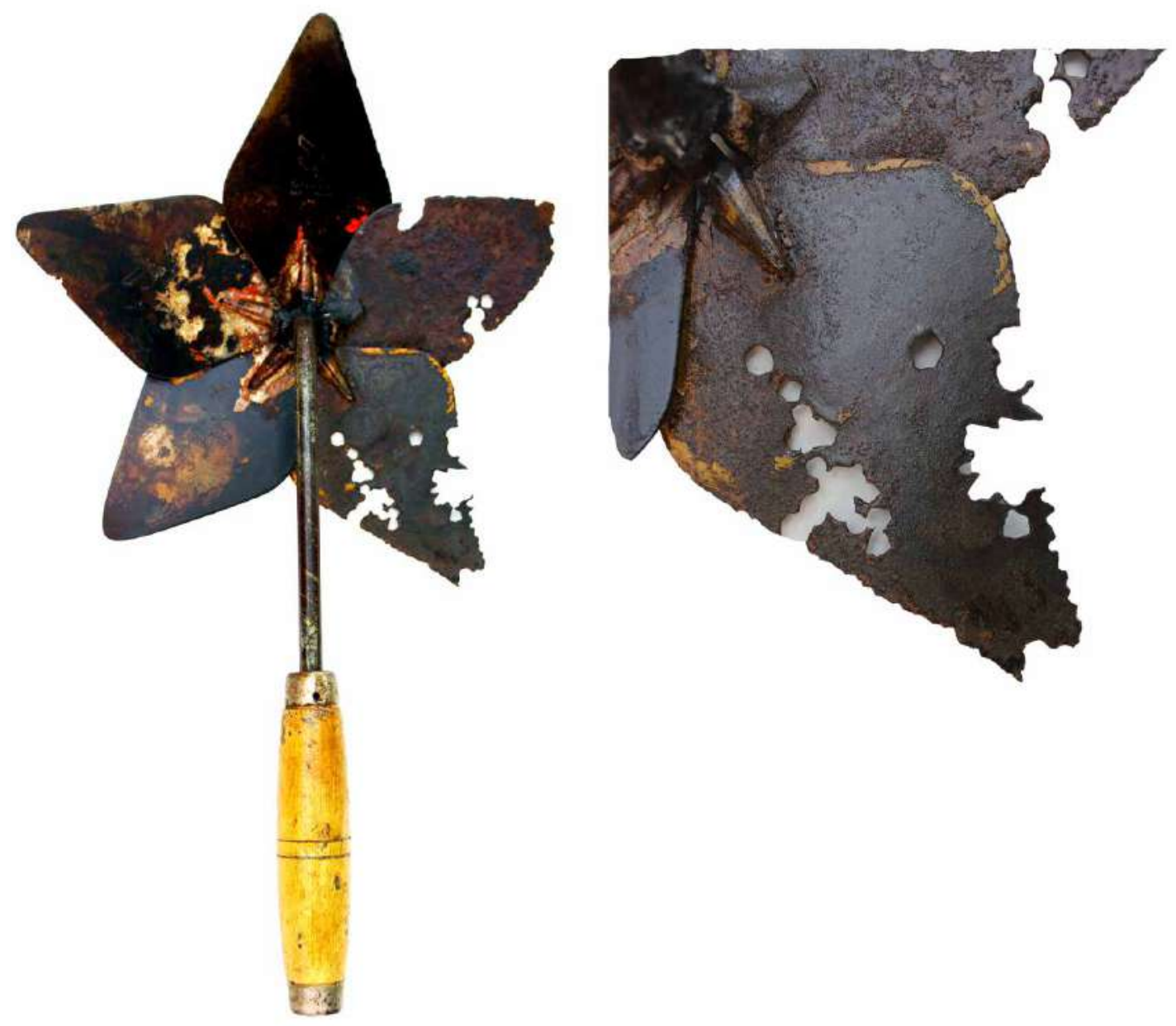

Yamith Quiroz David

Nuevas vegetaciones I

De la serie El mito de flora

Escultura, palustres, cabo de madera

$40,5 \times 24 \times 10 \mathrm{~cm}$

2020

Medellín 


\section{El análisis político transaccional de Oliver Williamson. In memoriam}

El 21 de mayo de 2020, en medio de la crisis mundial de la salud por el Covid-19, murió en Estados Unidos Oliver Williamson como consecuencia de una neumonía complicada por problemas de salud previos. Este científico social recibió en 2009, en compañía de la politóloga estadunidense Elinor Ostrom (1933-2012), el premio Nobel de Economía, en reconocimiento «por sus teorías sobre el papel de las empresas en la resolución de conflictos y por el análisis del papel de las empresas como estructuras de gobierno alternativas y sus límites» (The Nobel Prize, s. f.).

Oliver Williamson, quien es un autor poco conocido dentro de la ciencia política, realizó importantes aportes teóricos a este campo de estudio. Williamson es el artífice del enfoque análisis político transaccional (APT). El presente texto quiere destacar la propuesta teórica y analítica que hace este autor a la ciencia política contemporánea. Para esto se contextualizará la aparición de la propuesta dentro de los institucionalismos en las ciencias sociales, luego se exponen los rasgos generales del APT y, finalmente, la manera como su trabajo se viene utilizando y expandiendo en variados temas de los estudios políticos.

\section{Los nuevos institucionalismos en la ciencia política}

En 1989, cuando se publica El redescubrimiento de las instituciones. La base organizativa de la política (March y Olsen, 1997), el camino recorrido por los nuevos institucionalismos en ciencia política apenas iniciaba (Moe, 1990). En aquel momento el rol protagónico, sin duda, lo tenía el nuevo institucionalismo sociológico (Brinton y Nee, 1998; Romero, 1999). Sin embargo, en poco menos de una década y debido a los trabajos de autores como Thráinn Eggertsson (1995), Peter Hall y Rosemary Taylor (1996), Douglass North (1990a), Guy Peters (2003), Kenneth Shepsle (1999) y Peter Evans, Dietrich Rueschemeyer y Theda Skocpol (1985) se fue consolidando dentro de la ciencia política otros nuevos institucionalismos, entre ellos, el histórico y el de la elección racional (Hall y Taylor, 1996), para finalmente, al iniciar el nuevo siglo, tener como resultado al menos siete u ocho vertientes 
más de este importante enfoque dentro de nuestro campo disciplinar (Scott, 2001; Peters, 2003).

Sin embargo, desde 2003 la tendencia creciente de aparición de nuevos institucionalismos en ciencia política se ha reducido (Basabe, 2007; Eslava, Orozco y Valencia, 2011). Aunque se reconoce el apogeo y fortalecimiento constante que tiene este enfoque en la política, pues se sigue hablando de un «retorno de las instituciones» en la agenda de investigación principal de la ciencia política (Abal, 2012, pp. 55-59), al mismo tiempo se advierte que en lugar de la proliferación de un mayor número de enfoques de los nuevos institucionalismos lo que se viene produciendo es un diálogo y acompañamiento explicativo entre ellos.

En este escenario es que emerge la propuesta teórica de Oliver Williamson a los estudios políticos. El APT se presenta como una herramienta teórica que pueden utilizar los estudiosos de la política para analizar la realidad social. Es un enfoque que rescata los clásicos estudios del Estado y de la cultura política, e intenta inyectar mayor realismo a la explicación y comprensión de una amplia cantidad de fenómenos políticos (Evans, Rueschemeyer y Skocpol, 1985). Se caracterizan por defender a las instituciones como un rasgo estructural de la sociedad y como forma de gobierno que permanece en el tiempo y que afecta el comportamiento de los agentes políticos (Peters, 2003).

En este sentido, Williamson no continúa con la lógica de dividir los institucionalismos, sino de integrarlos, busca establecer un diálogo con los distintos enfoques, con el institucionalismo de la elección racional, con el histórico y con el económico, entre otros. Retoma del enfoque de la elección racional la idea de actores racionales y modelos subjetivos de análisis en la toma de decisiones políticas —individualismo metodológico-; del histórico la importancia del pasado en la comprensión de las ideologías, cuya idea se sintetiza en el concepto de senda o camino de la dependencia - path dependence o path dependency-, las cuales advierten de los cambios lentos que dan en las instituciones; y finalmente, del nuevo institucionalismo económico la idea de diversos mecanismos de coordinación y la importancia de los costos y de la información en las transacciones de diverso tipo.

En síntesis, el APT debe pensarse como un esfuerzo multidisciplinar que busca integrar enfoques, perspectivas de análisis y aportes variados; un 
programa de investigación que reconoce las contribuciones procedentes de la ciencia política, de la económica política y de la sociología política, además de otras de disciplinas como la ciencia cognitiva y la historia, y logra construir y proponer una visión de conjunto; un programa que enfatiza en el matrimonio que hay entre la teoría política y las ciencias sociales, intentando proponer y construir un programa de investigación más completo, realista e integrador, cuyo objetivo final es construir una visión funcional para el análisis de distintos fenómenos políticos (Lane y Ersson, 2000).

\section{Rasgos generales del análisis político transaccional}

Para el APT el mundo político es un universo de transacciones (North, 1998). Para satisfacer sus necesidades en el sistema político, los individuos deben realizar un gran conjunto de transacciones de todo tipo -opinar, participar, votar, marchar, protestar, entre otros-. Estas pueden ir desde la simple decisión de comprar un periódico para informarse sobre la propuesta política que hace un candidato a un cargo público, hasta colocarse en la posición de candidato y verse abocado a realizar un sinnúmero de transacciones para conseguir el objetivo de ser elegido, por ejemplo, inscribir su candidatura ante una autoridad, conseguir apoyos y recursos económicos para su campaña, o reunirse con los votantes en un gran escenario al cierre de su campaña. De allí que para el APT la unidad básica o más simple de análisis sea la transacción.

Para este enfoque, una transacción puede identificarse fácilmente, lo único que hay que hacer es observar analíticamente si es posible separar tecnológicamente una actividad de otra: «ocurre una transacción cuando se transfiere un bien o servicio a través de una interfaz tecnológicamente separable. Termina una etapa de la actividad y se inicia otra» (Williamson, 1989, p. 13). Lo que lleva a pensar la realización de un fin en una cadena amplia de actividades.

Así —continuando con el ejemplo del candidato político— es muy fácil separar la actividad de inscripción de candidatura ante una autoridad de otra donde se recolectan recursos para la campaña política, reconociendo que las dos transacciones hacen parte de una misma empresa que busca llevar a una persona a ocupar un cargo público mediante el voto popular. En la primera transacción los insumos y procesos son distintos a los utilizados en la segunda transacción. Lo que plantea el APT es que cualquiera sea la naturaleza del 
bien o servicio puede analizársele como una ocurrencia de transacciones entrelazadas —interfaces_-: cuando termina una etapa inicia otra que es tecnológicamente distinta.

Una vez identificada la transacción, el modelo advierte que su realización conlleva costos — costos de transacción—, idea proveniente de Ronald Coase (1937, p. 390), quien los define como aquellos en los que incurren los agentes — ciudadanos, organizaciones, entre otros - al hacer intercambios. En ellos se pueden incluir los costos de conseguir información - por ejemplo, en los que se incurre a la hora de conocer un programa político de un candidato-, de negociar y elaborar un contrato - por ejemplo, en el diseño y discusión de una norma local o nacional, tipo constitución- o de construir una norma formal y hacerla cumplir — por ejemplo, el análisis de ciclo de una política pública, desde su discusión normativa hasta su etapa de evaluación-. En síntesis, son los costos en que se incurre para administrar y hacer funcionar el sistema político.

Los costos de transacción surgen debido a tres actividades básicas: i) la planificación: se requiere planear el futuro y los individuos se ven limitados por la racionalidad, el mundo es complejo y hay incertidumbre que hace imprevisible el mundo que les rodea, esto significa que se requiere conocimientos que ayuden a predecir y planificar las contingencias (Simon, 1957); ii) los contratos son incompletos: no es posible hacer una planificación completa de los contratos, es difícil describir de manera minuciosa las acciones y además los estados posibles del mundo (Hart, 1995, p. 4); ii) es necesario hacer cumplir el contrato: existe contingencias donde puede no haber acuerdos en el desarrollo del contrato que hace que una tercera persona desinformada - por ejemplo, un tribunal — tenga que intervenir para hacer cumplir razonablemente. De allí que Williamson identifique los costos de transacción como los «costos comparativos de la planificación, la adaptación y el monitoreo de la terminación de la tarea bajo diversas estructuras de gobernación» (Williamson, 1989, p. 13). Costos que pueden incrementarse cuando las cosas no salen como se planeaba.

Para Williamson, en los sistemas políticos se presentan fricciones o fallas en el funcionamiento que provocan un incremento de los costos, debido al ambiente exógeno, no controlado. Las fricciones afectan al sistema político, reducen su normal funcionamiento y producen perturbaciones que no permiten el logro del objetivo, incluso pueden producir fallos en el 
sistema (Hardt, 2009). Lo que hacer el APT es identificar estas fricciones y encontrar las razones que las causan, a fin de corregirlas y reducir los costos. En otras palabras, el APT intenta conocer el funcionamiento de la maquinaria del sistema político, identificar y atender las fricciones y corregirlas para que finalmente se logre el objetivo. Estas fricciones son mitigadas en la medida en que las partes incurren en ciertos tipos de costos en cada una de las transacciones, garantizando que la transferencia funcione armoniosamente si se incide en costos asociados a la planeación, adaptación y monitoreo —bajo diversas estructuras de gobernación- de lo acordado.

Finalmente, el APT presenta, identifica y analiza las estructuras de gobernación. La gobernación, nos dice Williamson (2001, p. 12), es «un esfuerzo para crear orden y, por ende, mitigar el conflicto y lograr ganancias mutuas». Reconoce que los actores políticos cuentan con varias opciones para realizar los intercambios políticos u opciones de coordinación de las actividades, desde aquellas que consisten en dejar que otros decidan por ellos - que otros voten, que otros marchen o que otros hagan-, hasta organizarse para el hacerlo ellos mismos — yo decido, yo participo, yo opino-, incluso unirse con otras personas para lograr los fines —nosotros lo hacemos, nosotros participamos, nosotros opinamos-.

Cada una de estas formas de alcanzar los fines tienen asociadas alguna forma de gobierno o estructura de gobernanza. Lo que hace el modelo analítico transaccional es identificar las estructuras de gobierno y analizar en cada una de ellas los atributos básicos de la transacción: la incertidumbre, la frecuencia de las transacciones y la especificidad de los activos. El primer atributo se asocia al grado de incertidumbre - política- en el que se realiza la transacción; el segundo, a la recurrencia o regularidad con que los actores realizan las actividades asociadas al bien o servicio; y el tercero, a la especificidad del activo humano, es decir, al grado de conocimiento y especialización de los hombres y mujeres que se encargan de realizar las actividades políticas (Valencia, 2019).

Una vez analizado cada uno de estos atributos, en un contexto institucional —normas de juego-y organizativo — jugadores o actores-, la teoría permite sugerir una forma de gobierno para la consecución de la transacción. Estas estructuras de gobernación se mueven entre integración vertical o jerarquía unilateral, hasta opciones de mercado y multilateral. La elección de opción de gobierno más conveniente dependerá de la 
evaluación que se haga de los tres atributos. Así, por ejemplo, ante un grado de incertidumbre bajo y una especificidad de los activos alta, la teoría transaccional sugiere como opción de realización de la transacción la jerarquía u organización interna (Valencia, 2019).

En síntesis, el trabajo investigativo necesita identificar la transacción, designar y explicar los principales atributos de la transacción a fin de identificar los costos de transacción - costos de información, de contratación, de organización o burocracia, y de vigilancia de contratos, entre otros(Williamson, 1991, p. 13), para finalmente indicar la estructura de gobernanza - governance - más conveniente para promover la adaptación de naturaleza autónoma y cooperadora, todo ello en un contexto institucional y organizativo, y con presencia de agentes con racionalidad limitada y oportunistas.

\section{La agenda de investigación del análisis político transaccional en la ciencia política}

Al comenzar el nuevo milenio Williamson (2001) se puso a la tarea de hacer un balance sobre los nuevos institucionalismos y diseñó un esquema de síntesis donde ubica en niveles todo el programa de investigación de los nuevos institucionalismos. El autor identifica cuatro niveles de análisis con los que presenta un panorama global y muy completo para ubicar a los analistas y su trabajo científico:

El primer nivel corresponde a la teoría social desarrollada básicamente por los análisis históricos o de enraizamiento. Esta primera esfera se encarga de estudiar las instituciones formales e informales, las costumbres y tradiciones e, incluso, la religión. Es un análisis que se ocupa de trabajar aquellos fenómenos de largo plazo, como las tradiciones políticas, la cultura política y los cambios constitucionales (Caballero, 2004; North, 1984). Hacen parte de este nivel tabús, sanciones sociales, códigos de conducta y tradiciones, por ejemplo, las instituciones informales que se crean en torno a las religiones (Eslava, Orozco y Valencia, 2011). Así, si el interés es el análisis de dinámicas históricas y los cambios de larga duración en el sistema económico, cultural o político, el trabajo se situará en el nivel uno, sobre todo, los análisis políticos que se dirigen a analizar un primer momento histórico, aquel donde se toma la decisión de establecer una norma, las cuales cambian muy lentamente (North, 1990a; 1999; Acemoglu y Robinson, 2012). Mark Granovetter (1985) ha llamado a este comportamiento institucional de la sociedad como un «enraizamiento» 
social —social embeddedness—, que es cultural y político, y que hace parte de la estructura cultural de los pueblos (Williamson, 2001).

El segundo nivel corresponde propiamente al ambiente institucional, se centra en las reglas formales. Aunque también hace parte de la estructura cultural de la población, se diferencia del anterior nivel en el sentido de que permite cambios o rediseños institucionales. Permite, al contrario del nivel uno, las elecciones institucionales calculadas. Estudia el ambiente institucional de mediano plazo, las reglas formales del juego y la organización política, el funcionamiento del poder público y la burocracia (Caballero, 2006; 2008). Son trabajos que intentan analizar y establecer el ambiente institucional idóneo y la economización de costos de transacción de primer orden, y hacen parte de la denominada teoría política positiva. Entre sus temas están todos los estudios sobre los derechos de propiedad, al igual que los que hace la economía política positiva (Buchanan, 1984; Hirschman, 1989). Hacen parte de este nivel las constituciones, las leyes, los decretos y normas legales (North, 1984; Tsebelis, 1990). Se encargan del diseño de este tipo de instituciones los poderes del Estado, como el Ejecutivo, el Legislativo y el Judicial (Weingast y Marshall, 1988; Caballero, 2007); también la burocracia, tanto central como descentralizada (Caballero, 2006, 2008). Williamson ubica a la economía de los derechos de propiedad en este segundo nivel y lo plantea como de economización porque trata de establecer los derechos de propiedad sobre los recursos en un primer plano, es decir, las reglas formales del juego que los individuos configuran para organizar la sociedad — por ejemplo, las Constituciones-. Allí se establece un sistema de leyes que regulan los derechos que los individuos tienen sobre sus propiedades (Demsetz, 1969; 1982).

El tercer nivel de análisis, denominado de gobernación o de las estructuras de governance, se relaciona con la gobernación de las relaciones contractuales, lo que lleva a ocuparse de sus instituciones. El objetivo es establecer estructuras de gobernación idóneas buscando economizar costos de transacción de segundo orden y para esto analiza las reglas de juego, los contratos y el alineamiento de las estructuras de gobernación con las transacciones. Los análisis de este nivel se concentran en alinear las estructuras de gobernación de acuerdo con el tipo de transacciones que realizan los agentes a fin de alcanzar la cooperación y la adaptabilidad. Según Williamson (2001, p. 13), estas estructuras de gobernación y su posible «reorganización de las transacciones entre las estructuras de gobernación se reexamina 
periódicamente, en el orden de un año a una década, a menudo coincidiendo con los intervalos de renovación de los contratos o de los equipos».

Los trabajos sobre este nivel de análisis comenzaron en la década de 1970 con los primeros escritos de Williamson y continuó hasta su muerte en 2020 (1971; 1976; 1979; 1985; 1991; 1999; 2003; 2010). El interés aquí no es el sistema legal como en el segundo nivel, que establezca las normas y vele por su cumplimiento, lo importante son las estructuras de gobernación (Campbell, Hollingsworth y Lindberg, 1991; Dixit, 2009; 1998; Moe, 1997). Igualmente, en el avance hacia la eficiencia institucional desempeña un papel central la noción de compromiso, «lo que justifica la importancia del capital reputacional y de las fórmulas organizativas del Estado» (Arias y Caballero, 2004, pp. 8-9). Es un ordenamiento público-privado basado en las normas más generales que actúan en este escenario, pero con discrecionalidad para hacer nuevos acuerdos. Se aceptan las normas generales plurales y se elaboran las normas particulares y singulares (MacNeil, 1974; Summers, 1969).

Finalmente, el nivel interno de las organizaciones, en el que se analiza en el momento presente la manera como se da la distribución y empleo de recursos, a la vez que se identifican los costos en que incurren los agentes al realizar transacciones. Es un análisis microfundamentado, marginal y basado en la idea de función de producción, en el cual se busca la optimalidad, con el objetivo de establecer condiciones marginales idóneas, alineamiento de incentivos y la economización de tercer orden. En este nivel se investiga la realización de teorías como la de agencia o agente-principal, la cual se ocupa de los alineamientos ex-ante de los incentivos, dejando de lado la contratación ex-post y el manejo eficiente de los riesgos (Aoki, 2001; González, 2014; Jensen y Meckling, 1994). Unas enfatizan en el comportamiento de las organizaciones — políticas-, ven a los partidos políticos o al Estado como empresas en un escenario de juego de coaliciones entre agentes, otras analizan a la organización política internamente y la manera como se persigue objetivos, se delega actividades y se controlan e incentivan conductas en los agentes — teoría de agencia- (Jensen y Meckling, 1994). En estos análisis cumple un papel muy importante la teoría de incentivos y las que hablan de división y especialización del trabajo. En el APT, un ejemplo es el estudio de los efectos que tiene en un alzado en armas un incentivo económico para la dejación de armas (Valencia y Bedoya, 2015; Hincapié y Valencia, 2014). 
Es necesario advertir que, a pesar de la diferenciación y separación aparente entre cada uno de los niveles de análisis, Williamson (2001) insiste en que los niveles están interconectados y relacionados. El componente institucional cumple el papel de unir los diversos niveles de análisis: las instituciones funcionan como normas que establecen restricciones a todos y cada uno de los niveles. Se produce una especie de feedback donde un nivel — superior o inferior — afecta al siguiente nivel — superior o inferior-. Allí caben todos los temas y problemas del APT, desde el histórico y de largo plazo que realiza la historia política, hasta el coyuntural y de plazo inmediato de los modelos de elección racional. Lo que hace la división en niveles es permitir identificar intereses específicos de cada línea de trabajo y que según el foco de trabajo el analista político pueda situarse en la larga duración o en un momento específico de la historia para estudiar la coyuntura.

En conclusión, el APT es un enfoque, una perspectiva analítica que convoca a los politólogos a abordar temas diversos (Arias y Caballero, 2013a; 2013b; Caballero, 2002a; 2002b; 2007; 2011; North, 1990b), es una perspectiva de análisis creada y desarrollada en las últimas cuatro décadas y que viene conformándose en un gran programa de investigación científica (Williamson, 2010). Es una agenda de investigación con una disparidad de intereses en variados temas que ha hecho posible que surjan líneas de investigación abundantes (Dixit, 1996; North, 1990a), ofreciendo una propuesta analítica que puede ser aplicada con toda propiedad en el estudio de fenómenos políticos. Unos se ocupan de ir al pasado, de subirse a la montaña y ver en el horizonte todo el territorio; otros, en cambio, se interesarán por ir a los lugares públicos y al ámbito de lo privado, no suben a la montaña, se quedan en los espacios de interacción humana analizando a las organizaciones políticas en su interior.

\section{Germán Darío Valencia Agudelo (Colombia)*}

\footnotetext{
* Director revista Estudios Políticos. Economista. Especialista en Gerencia Social. Magíster en Ciencia Política. Doctor en Estudios Políticos. Grupo Hegemonía, guerras y conflictos, Instituto de Estudios Políticos, Universidad de Antioquia UdeA. Calle 70 No. 52-21, Medellín, Colombia. Correo electrónico: german.valencia@udea.edu.co - Orcid: 0000-0002-6412-6986 - Google Scholar https:// scholar.google.es/citations?user=7Sm8z3MAAAAJ\&hl=es
}

Cómo citar este artículo: Valencia Agudelo, Germán Darío. (2020). Editorial. El análisis político transaccional de Oliver Williamson. In memoriam. Estudios Políticos (Universidad de Antioquia), 59, pp. 9-22. DOI: 10.17533/udea.espo.n59a01 


\section{Referencias bibliográficas}

1. Abal Medina, Juan Manuel. (2012). Manual de ciencia política. Buenos Aires: Eudeba.

2. Acemoglu, Daron y Robinson, James. (2012). Por qué fracasan los países. Barcelona: Dausto.

3. Aoki, Masahiko. (2001). Toward a Comparative Institutional Analysis. Cambridge: MIT. https://doi.org/10.7551/mitpress/6867.001.0001

4. Arias, Xosé Carlos y Caballero, Gonzalo. (2004). El retorno de las instituciones y la teoría de la política económica: introducción y revisión de la literatura. Papeles de Trabajo, Instituto de Estudios Económicos de Galicia, 70, Recuperado de https:// economica09.files.wordpress.com/2009/07/arias-y-caballero_el-retorno-de-lasinstituciones.pdf

5. Arias, Xosé Carlos y Caballero, Gonzalo. (2013a). Instituciones, costos de transacción y políticas públicas: un panorama. Revista de Economía Institucional, 5 (8), pp. 117-146.

6. Arias, Xosé Carlos y Caballero, Gonzalo (eds.). (2013b). Nuevo institucionalismo: gobernanza, economía y políticas públicas. Madrid: Centro de Investigaciones Sociológicas.

7. Basabe, Santiago. (comp.). (2007). El nuevo institucionalismo en ciencia política: perspectivas, enfoques y campos de acción. En: Instituciones e institucionalismo en América Latina (pp. 173-202). Quito: Cipec.

8. Brinton, Maty \& Nee, Victor (eds.). (1998). The New Institutionalism in Sociology. New York: Russell Sage Foundation.

9. Buchanan, James. (1984). Política sin romanticismos. Esbozo de una teoría positiva de la elección pública y de sus implicancias normativas. En: Buchanan, James; McCormick, Robert \& Tollison, Robert (eds.). El análisis económico de lo político. Madrid: Instituto de Estudios Económicos.

10. Caballero, Gonzalo. (2002a). El programa de la nueva economía institucional: lo macro, lo micro y lo político. Ekonomiaz, 50, pp. 230-261.

11. Caballero, Gonzalo. (2002b). La economía regional de los costes de transacción: un enfoque neoinstitucional. Revista Asturiana de Economía, 25, pp. 167-186.

12. Caballero, Gonzalo. (2004). Instituciones e historia económica: enfoques y teorías institucionales. Revista de Economía Institucional, 10, pp. 137-157.

13. Caballero, Gonzalo. (2006). Abriendo la caja negra del Estado español: reglas, vetos, intereses y jerarquía en la formación de la política económica. Problemas del Desarrollo, 37 (144), pp. 192-219. https://doi.org/10.22201/ iiec.20078951e.2006.144.7603

14. Caballero, Gonzalo. (2007). Nuevo institucionalismo en ciencia política, institucionalismo de elección racional y análisis político de costes de transacción: 
una primera aproximación. Revista de Investigaciones Políticas y Sociológicas, 6 (2), pp. 9-27.

15. Caballero, Gonzalo. (2008). El cambio institucional de la economía española del franquismo a la democracia. Un análisis histórico institucional. Política y Gobierno, xv (2), pp. 353-401.

16. Caballero, Gonzalo. (2011). Economía de las instituciones: de Coase y North a Williamson y Ostrom. Ekonomiaz, 77 (2), pp. 14-51.

17. Campbell, John; Hollingsworth, Rogers \& Lindberg, Leon. (1991). Governance of the American Economy. Cambridge: Cambridge University. https:// doi.org/10.1017/СBO9780511664083

18. Coase, Ronald. (1937). The Nature of the Firm. Economica, vi, pp. 386-405. https://doi.org/10.1111/j.1468-0335.1937.tb00002.x

19. Demsetz, Harold. (1969). Information and Efficiency: Another Viewpoint. Joural Law Economic, 12 (1), pp. 1-22. https://doi.org/10.1086/466657

20. Demsetz, Harold. (1982). The Structure of Ownership and the Theory of the Firm. The Journal of Law y Economics, 26 (2), pp. 375-390. https://doi. org/10.1086/467041

21. Dixit, Avinash. (1996). The Making of Economic Policy: A Transaction-Cost Politics Perspective. Boston: MIT. https://doi.org/10.7551/mitpress/4391.001.0001

22. Dixit, Avinash. (1998). Transaction Cost Politics: A Framework and a Case Study. In: Baldassarri, Mario; Paganetto, Luigi \& Phelps, Edmund (eds.). Institutions and Economic Organization in the Advanced Economies: The Governance Perspective (Central Issues in Contemporary Economic Theory and Policy). Nueva York: St. Martin's. https://doi.org/10.1007/978-1-349-26956-3_7

23. Dixit, Avinash. (2009). Governance Institutions and Economic Activity. American Economic Review, 99 (1), pp. 3-24. https://doi.org/10.1257/aer.99.1.5

24. Eggertsson, Thráinn. (1995). El comportamiento económico de las instituciones. Madrid: Alianza.

25. Eslava, Adolfo; Orozco, Hernán y Valencia, Germán. (2011). Los nuevos institucionalismos como riqueza metodológica para el estudio de la política. Opera, 11 , pp. 5-28.

26. Evans, Peter; Rueschemeyer, Dietrich \& Skocpol, Theda. (1985). Bringing the State Back In. Cambridge: Cambridge University. https://doi.org/10.1017/ CBO9780511628283

27. González-Chavarría, Alexander. (2014). Análisis institucional multiagente: el problema de estructuración y agencia en la explicación de la emergencia de estructuras de gobernanza. Revista de Estudios Sociales, 49, pp. 190-204. https://doi. org/10.7440/res49.2014.14

28. Granovetter, Mark. (1985). Economic Action and Social Structure: The Problem of Embeddedness. American Journal of Sociology, 91 (3), pp. 481-510. https://doi.org/10.1086/228311 
29. Hall, Peter \& Taylor, Rosemary. (1996). Political Science and the Three New Institutionalisms. Political Studies, xuIv, pp. 936-957. https://doi. org/10.1111/j.1467-9248.1996.tb00343.x

30. Hardt, Łukasz. (2009). The History of Transaction Cost Economics and its Recent Developments. Erasmus Journal for Philosophy and Economics, 2 (1), pp. 2951. https://doi.org/10.23941/ejpe.v2i1.22

31. Hart, Oliver. (1995). Firms, Contracts, and Financial Structure. Oxford: Oxford University. https://doi.org/10.1093/0198288816.001.0001

32. Hincapié, Sindy y Valencia Germán. (2014). Incentivos económicos para la desmovilización de la insurgencia en Colombia. Perfil de Coyuntura Económica, 24, pp. 49-69. https://doi.org/10.17533/udea.pece.n24a03

33. Hirschman, Albert. (1989). Enfoques alternativos sobre la sociedad de mercado y otros ensayos recientes. México, D. F.: Fondo de Cultura Económica.

34. Jensen, Michael y Meckling, William. (1994). Teoría de la empresa: la gerencia costes de agencia y estructura de la propiedad. En: Putterman, Louis (ed.). En la naturaleza económica de la empresa (pp. 261-286). Madrid: Alianza.

35.Lane,Jan-Erik\&Ersson,Svante.(2000). TheNewInstitutionalPolitics. Performance and Outcomes. London: Routledge. https://doi.org/10.4324/9780203264607

36. MacNeil, lan. (1974.). The Many Futures of Contracts. Southern California Law Review, 47 (2), pp. 691-816.

37. March, James y Olsen, Johan. (1997). El redescubrimiento de las instituciones. La base organizativa de la política. México, D. F.: Colegio Nacional de Ciencias Políticas y Administración Pública, Universidad Autónoma de Sinaloa, Fondo de Cultura Económica.

38. Moe, Terry. (1990). Political Institutions: The Neglected Side of the Story. Journal of Law, Economics and Organization, 6, pp. 213-253. https://doi.org/10.1093/ jleo/6.special_issue.213

39. Moe, Terry. (1997). The Positive Theory of Public Bureaucracy. En: Mueller, Dennis (ed.). Perspectives on Public Choice (pp. 455-480). New York: Cambridge University. https://doi.org/10.1017/CBO9780511664458.022

40. North, Douglass. (1984). Estructura y cambio en la historia económica. Madrid: Alianza.

41. North, Douglass. (1990a). Instituciones, cambio institucional y desempeño económico. México, D. F.: Fondo de Cultura Económica.

42. North, Douglass. (1990b). A Transaction Cost Theory of Politics. Journal of TheoreticalPolitics, 2(4),pp.355-367.https://doi.org/10.1177/0951692890002004001

43. North, Douglass. (1998). Una teoría de la política basada en el enfoque de los costos de transacción. En: Saiegh, S. y Tommasi, M. (comps.). La nueva economía política: Racionalidad e instituciones (pp. 97-112). Buenos Aires: Eudeba.

44. North, Douglass. (1999). In Anticipation of the Marriage of Political and Economic Theory. In: Alt, James; Levi, Margaret \& Ostrom, Elinor (eds.). Competition 
and Cooperation. Conversations with Nobelists about Economics and Political Science (pp. 197-252). New York: Russell Sage Foundation.

45. Peters, Guy. (2003). El nuevo institucionalismo. Teoría institucional en la ciencia política. Barcelona: Gedisa.

46. Romero, Jorge. (1999). Estudio introductorio. Los nuevos institutucionalismos: sus diferencias, sus cercanias. En: Powell, Walter y DiMaggio, Paul (eds.). El nuevo institucionalismo en el análisis organizacional (pp. 7-29). México, D. F.: Colegio Nacional de Ciencias Políticas y Administración Pública, Universidad Autónoma del Estado de México, Fondo de Cultura Económica.

47. Scott, William. (2001). Institutions and Organizations. Thousand Oaks: Sage.

48. Shepsle, Kenneth. (1999). El estudio de las instituciones: lecciones del enfoque de la elección racional. En: Farr, James; Dryzek, John y Leonard, Stephen (eds.). La ciencia política en la historia (pp. 354-377). Madrid: Istmo.

49. Simon, Herbert. (1957). Models Of Man. New York: John Wiley.

50. Summers, Clyde. (1969). Collective Agreements and the Law of Contracts. Yale Law Journal, 78 (4), pp. 525-575. https://doi.org/10.2307/795018

51. The Nobel Prize. (s. f.). The Sveriges Riksbank Prize in Economic Sciences in Memory of Alfred Nobel 1978 Herbert A. Simon. Recuperado de http://www. nobelprize.org/nobel_prizes/economic-sciences/laureates/1978/

52. Tsebelis, George. (1990). Nested Games: Rational Choice in Comparative Politics. Berkeley: University of California. https://doi.org/10.1525/9780520911970

53. Valencia, Germán. (2019). Organizarse para negociar la paz. Medellín: Universidad de Antioquia.

54. Valencia, Germán y Bedoya, Claudia. (2015). Transacciones, fricciones y contratos en las negociaciones de paz con las FARC, 2010-2016. Perfil de Coyuntura Económica, 26, pp. 7-41. https://doi.org/10.17533/udea.pece.n26a01

55. Weingast, Barry \& Marshall, William. (1988). The Industrial Organization of Congress; or, Why Legislatures, Like Firms, Are Not Organized as Markets. Journal of Political Economy, 96 (1), pp. 132-163. https://doi.org/10.1086/261528

56. Williamson, Oliver. (1971). The Vertical Integration of Production: Market Failure Considerations. American Economic Review, 61 (2), pp. 112-123.

57. Williamson, Oliver. (1976). Franchise Bidding for Natural Monopolies-In General and with Respect to CATV. The Bell Journal of Economics, 7 (1), pp. 73-104. https://doi.org/10.2307/3003191

58. Williamson, Oliver. (1979). Transaction-Cost Economics: The Governance of Contractual Relations. The Journal of Law and Economics, 22 (2), pp. 233-261. https:// doi.org/10.1086/466942

59. Williamson, Oliver. (1985). Reflections on the New Institutional Economics. Journal of Institutional and Theoretical Economics, 141 (1), pp. 187-95.

60. Williamson, Oliver. (1989). Las instituciones económicas del capitalismo. México, D. F.: Fondo de Cultura Económica. 
61. Williamson, Oliver. (1991). Comparative Economic Organization: The Analysis of Discrete Structural Alternatives. Administrative Science Quarterly, 36 (2), pp. 269-296. https://doi.org/10.2307/2393356

62. Williamson, Oliver. (1999). Public and Private Bureaucracies: A Transaction Cost Economics Perspective. The Journal of Law, Economics, \& Organization, 15 (1), pp. 306-342. https://doi.org/10.1093/jleo/15.1.306

63. Williamson, Oliver. (2001). La nueva economía institucional: balance y perspectivas. Revista $B C V, 15$ (1), pp. 6-34.

64. Williamson, Oliver. (2003). Examining Economic Organization Through the Lens of Contract. Industrial and Corporate Change, 12 (4), pp. 917-942. https://doi. org/10.1093/icc/12.4.917

65. Williamson, Oliver. (2010). Transaction Cost Economics: The Natural Progression. The American Economic Review, 100 (3), pp. 673-690. https://doi. org/10.1257/aer.100.3.673 